Variations sur la lettre, le mètre et la mesure :

Shakespeare

\title{
Le théâtre et l'invention de la lecture silencieuse en Grèce ancienne
}

Jesper Svenbro

\section{(2) OpenEdition \\ Journals}

\section{Édition électronique}

URL : http://journals.openedition.org/shakespeare/984

DOI : 10.4000/shakespeare.984

ISSN : 2271-6424

Éditeur

Société Française Shakespeare

Édition imprimée

Date de publication : 1 novembre 1996

Pagination : 177-186

\section{Référence électronique}

Jesper Svenbro, «Le théâtre et l'invention de la lecture silencieuse en Grèce ancienne », Actes des congrès de la Société française Shakespeare [En ligne], 14 | 1996, mis en ligne le 01 janvier 2007, consulté le 20 avril 2019. URL : http://journals.openedition.org/shakespeare/984 ; DOI : 10.4000/ shakespeare.984 


\section{Variations sur la lettre,}

le mètre et la mesure

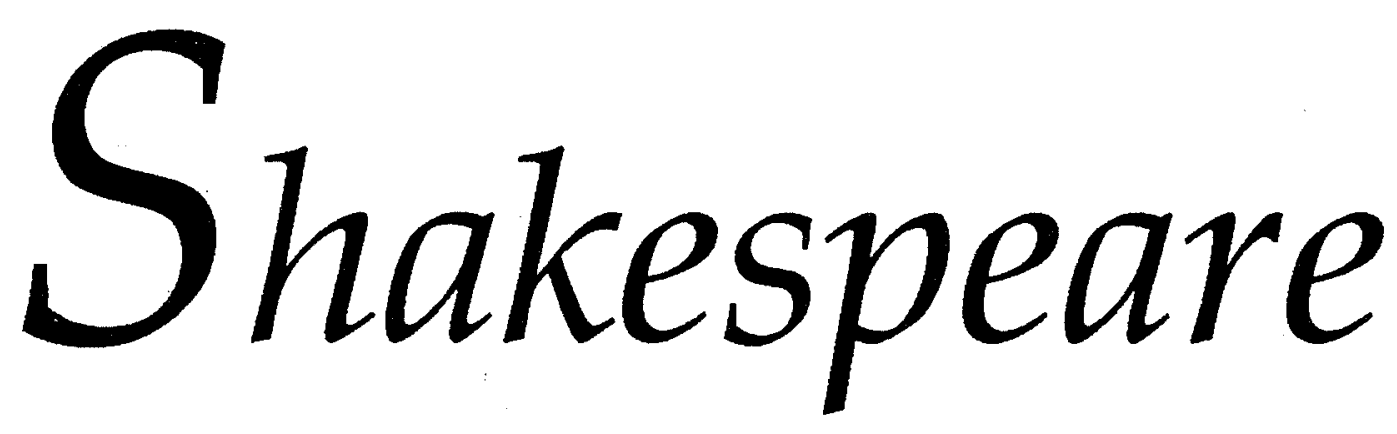

textes présentés par Dominique Goy-Blanquet

Directeur de la publication : Richard Marienstras 


\section{Le théâtre et l'invention de la lecture silencieuse en Grèce ancienne ${ }^{1}$}

Dans son article "Silent Reading in Antiquity" de 1968, Bernard Knox cite deux textes du $\mathrm{V}^{\mathrm{e}}$ siècle av. J.-C. qui semblent montrer que - contrairement à ce qu'ont pu dire les philologues - les Grecs pratiquaient la lecture silencieuse et qu'à l'époque de la guerre de Péloponnèse les poètes dramatiques pouvaient compter sur une familiarité avec elle auprès de leur public ${ }^{2}$.

Le premier de ces textes est un passage de l'Hippolyte d'Euripide, datant de 428 , où Thésée aperçoit la tablette d'écriture qui pend de la main de Phèdre morte et se demande ce qu'elle peut bien vouloir lui annoncer. Il rompt le sceau. Le chœur intervient pour chanter son inquiétude, jusqu'à ce qu'il soit interrompu par Thésée : "Hélas! Au malheur quel malheur vient s'ajouter, intolérable, indicible ! Infortuné que je suis !" s'exclame celui-ci. Sur la demande du chœur, il révèle ensuite le contenu de la tablette - non pas en la lisant à haute voix mais en résumant son contenu. Il l'a clairement lue en silence, pendant le chant du chœur ${ }^{3}$.

Le deuxième texte de Knox est tiré des Cavaliers d'Aristophane, qui date de 424. Deux personnages parlent d'un oracle écrit que Nicias a réussi à voler à un certain Paphlagon. "Apporte-le moi pour que je le lise", dit Démosthène à Nicias, qui lui remplit une première coupe de vin et demande : "Que dit l'oracle ?" Sur quoi Démosthène, absorbé dans sa lecture, lui répond : "Remplis-moi une autre coupe !" - "Dit-il vraiment 'Remplis-moi une autre coupe' ?" demande alors Nicias, persuadé que Démosthène fait une lecture à haute voix de l'oracle. Cette plaisanterie est reprise et développée dans les vers qui suivent, jusqu'à ce que Démosthène révèle à Nicias : "Là-dedans, il est dit comment Paphlagon va périr lui-même". Il donne ensuite un résumé de l'oracle. Il ne le lit pas : il l'a déjà fait en silence 4 .

Ce passage nous présente donc un lecteur habitué à lire de tête (il sait même demander à boire en le faisant !) à côté d'un auditeur qui ne semble point habitué à cette nouvelle pratique mais qui prend les paroles prononcées par le lecteur pour des paroles lues. Pour Nicias, "lecture" signifie en fait "lecture à haute voix", conformément à ce qui devait être la norme en Grèce ancienne ${ }^{5}$.

La scène des Cavaliers est particulièrement instructive, au moins de prime abord, car elle indique que la pratique de la lecture silencieuse n'est pas chose courante en 424 (Platon a alors cinq ans), même si elle est supposée connue du public de la comédie. C'est donc une pratique réservée à un nombre limité de lecteurs et inconnue à bon nombre de Grecs, surtout, peut-on penser, à ceux, analphabètes, qui ne connaissent l'écriture que de l' "extérieur", comme Nicias.

En outre, il faut rappeler que les deux documents cités sont de provenance athénienne : à des endroits où, comme à Sparte, on s'efforçait de limiter l'ensei- 
gnement des lettres au "strict nécessaire"6, la lecture silencieuse devait être encore moins susceptible d'être connue, voire pratiquée. Le lecteur "normal" lisait peu et de façon sporadique. Et toujours à haute voix.

Pour ce lecteur, c'est en fait la voix qui donne accès au sens de l'écrit. Plus exactement, la voix du lecteur est l'instrument par lequel la séquence graphique, écrite sans le signe intervalle, est reconnue comme du langage. L'absence du signe intervalle organise et structure cette lecture traditionnelle, en rendant quasi nécessaire l'intervention de la voix du lecteur. Quiconque a essayé de lire une page dactylographiée sans le signe intervalle, c'est-à-dire en scriptio continua, sait que la compréhension du texte tire le plus grand profit de sa vocalisation. La lecture à haute voix rend intelligible ce qui semble incompréhensible à l'œil. Et pourtant les Grecs ne se sont pas pressés d'introduire le signe intervalle dans l'écriture. Pourquoi ?

Dans la culture grecque, la mise en son de l'écrit est dès le début considérée comme une valeur en soi et, d'une certaine manière, comme le but même de l'écriture $^{7}$. L'écrit est là pour produire du son. Dans ces conditions, pourquoi vouloir lire en silence ? Pourquoi vouloir faciliter la lecture silencieuse en introduisant la séparation entre les mots ? Si la lecture silencieuse ne présente pas d'intérêt, pourquoi se soucier de l'absence du signe intervalle?

Mais il ne faut peut-être pas exagérer le poids de cette absence. En réalité, la scriptio continua n'a pas été un obstacle insurmontable à la nouvelle forme de lecture, comme on aurait pu le croire à partir de l'expérience médiévale : selon Paul Saenger, la word division a été une condition nécessaire pour que la lecture silencieuse - pratiquée par des moines copiant des textes en silence - puisse se répandre $^{8}$. Car si nous suivons l'interprétation de Bernard Knox, les anciens Grecs ont vraiment su lire en silence, tout en gardant leur scriptio continua.

Selon la suggestion de Knox, c'est la fréquentation de grandes quantités de textes qui a ouvert la possibilité d'une lecture silencieuse dans l'antiquité, silencieuse et donc rapide ${ }^{9}$. Au $\mathrm{V}^{\mathrm{e}}$ siècle, un Hérodote a vraisemblablement dû abandonner la lecture à haute voix au cours de son travail d'historien et, déjà pendant la deuxième moitié du VI $\mathrm{V}^{\mathrm{e}}$ siècle, ceux qui, dans un but quasi philologique, se sont occupés du texte homérique sous les Pisistratides à Athènes ont sans aucun doute eu l'occasion de développer cette technique. Technique réservée à une minorité, bien entendu, mais une minorité importante (dans laquelle se trouvent certainement les poètes dramatiques) et digne d'être étudiée le long de son histoire première.

La seule introduction du signe intervalle n'a pas suffi pour généraliser la lecture silencieuse au Moyen Age. Il a fallu plus que cette innovation technique faite déjà au VII ${ }^{\mathrm{e}}$ siècle de notre ère. Il a fallu les exigences de la science scolastique pour que les avantages de la lecture silencieuse - rapidité, intelligibilité soient découverts et exploités. Ce n'est en effet qu'au sein de la science scolastique que la lecture silencieuse a pu "prendre" - tout en restant pratiquement inconnue dans le reste de la société médiévale ${ }^{10}$.

De la même façon, dirais-je, la seule fréquentation de grandes quantités de textes n'est pas un facteur suffisant pour que la lecture silencieuse "prenne" au cours du V $\mathrm{V}^{\mathrm{e}}$ siècle avant notre ère dans certains milieux de la Grèce ancienne. La 
lecture extensive apparaît plutôt comme le résultat d'une innovation qualitative, dans l'attitude vis-à-vis de l'écrit. Comme le résultat de tout un cadre mental, nouveau et puissant, capable de restructurer les catégories de la lecture traditionnelle. La lecture silencieuse ne saurait être structurée par le seul fait quantitatif : à vrai dire, Knox lui-même ne cite que des auteurs post-classiques lorsqu'il veut évoquer les vastes lectures des anciens. Elle pourrait l'être en revanche, je pense, par l'expérience du théâtre.

Quels traits distinctifs de la représentation théâtrale sont assez nets et assez originaux pour avoir pu structurer la nouvelle pratique de la lecture silencieuse ? On pense d'abord, naturellement, à la séparation très marquée entre la scène et le public. Cette séparation délimite le jeu fictif qui se déroule sur scène et constitue, d'une certaine manière, l'originalité même du théâtre : le public ne peut pas intervenir dans ce jeu. Il ne peut pas, par exemple, communiquer à un personnage sur scène ce qu'il sait déjà sur son destin ${ }^{11}$. Il ne peut pas arrêter le cours des événements pour expliquer aux personnages ce qu'il faut faire. Il doit les "contempler" (theasthai) lorsque, dans le jeu tragique, ils vont vers leur propre destruction. La tension créée par cette situation rend l'action sur scène d'autant plus fascinante : le spectacle théâtral se livre dans une autonomie que le public ne doit pas déranger, ainsi que le veut la règle du "jeu" (paidia) dont parle Thespis, lorsqu'en plein VI siècle il défend son art nouveau contre la critique indignée de Solon ${ }^{12}$.

Le public - et déjà celui de Thespis - doit regarder et écouter. Passivement. Ce n'est pas aux spectateurs d'intervenir sur scène ni de lire le texte qui, absent de la scène, y régit néanmoins toute l'action. Mémorisé par les acteurs, le texte n'est pas visible au moment où il est dit $^{13}$ : les acteurs se sont substitués à lui, de façon à le traduire en "écriture vocale" (expression que je justifierai plus loin). Les acteurs ne le lisent pas : ils en produisent une copie vocale. En cela, ils se distinguent du lecteur ordinaire qui, aux yeux des Grecs, prête sa voix à l'écrit devant lui. Dans la lecture, il y a une proximité, voire une contiguïté, entre écriture et voix, dont la dernière est censée "prolonger" la première.

En revanche, la distance entre le texte dramatique et la diffusion qu'en assurent les acteurs paraît assez grande pour que cette diffusion soit qualifiée d'écriture vocale, distincte de la lecture. Avant le spectacle, les acteurs ont peut-être lu le texte pour le mémoriser mais, pendant la représentation, leurs voix se substitueront à l'écrit. Les spectateurs écouteront leur "écriture vocale". Et si l'acteur ne se confond pas avec le lecteur, l'écoute de cette écriture vocale ne fait pas des spectateurs des lecteurs traditionnels non plus. En tant que spectateurs, ils n'ont pas à activer ou à réactiver l'écrit par l'intervention de leur propre voix, car l'écrit leur parle en toute autonomie. Ils écoutent, passivement, une écriture. Une écriture vocale.

La séparation entre la scène, où cette écriture vocale est livrée, et le public qui écoute, est sans doute assez nette pour avoir suggéré aux Grecs une séparation analogue entre écrit et lecteur. Ou mieux : pour leur avoir ouvert la possibilité d'une nouvelle attitude vis-à-vis de l'écrit. Le lecteur traditionnel, qui a besoin de sa voix pour "reconnaître" (anagignôskein) la séquence graphique, entretient avec l'écrit, sur le plan de la mise en son, une relation sensiblement active. Il doit faire un effort mental et physique pour accomplir sa fonction instrumentale, sinon les 
lettres resteront vides de sens. Au contraire, celui qui sait lire en silence a un rapport à l'écrit qui apparaît plutôt comme passif. Il n'est plus l'instrument de l'écrit, car l'écrit lui "parle" tout seul. Au lecteur d' "écouter" passivement.

Disons plutôt : l'activité de celui qui lit en silence n'est pas vécue comme un effort pour déchiffrer, c'est une activité qui s'ignore comme telle (de même que l'activité interprétative de 1' "oreille" écoutant une séquence sonore signifiante est une activité qui s'ignore comme telle - elle apparât plutôt comme une réception passive). Dans la lecture silencieuse, la "reconnaissance" du sens est immédiate; elle n'est pas précédée d'un moment opaque. Le lecteur qui lit dans sa tête n'a pas à activer ou à réactiver l'écrit par l'intervention de sa voix. L'écriture lui semble tout simplement parler. Il est à l'écoute d'une écriture - de même que le spectateur au théâtre est à l'écoute de l'écriture vocale des acteurs. L'écrit qui est "reconnu" de façon visuelle semble posséder la même autonomie que le spectacle théâtral. Les lettres se lisent - ou plutôt se disent - elles-mêmes. Le lecteur "silencieux" n'a pas à intervenir sur la scène de l'écriture : capables de "parler", les lettres peuvent se passer de l'intervention de sa voix. Elles en possèdent une déjà. Et au lecteur simplement de l' "écouter" - à l'intérieur de lui-même. La voix lectrice se trouve intériorisée.

Si cette "passivité" du lecteur est l'héritière de la passivité du spectateur de théâtre, jusqu'à quel point, en remontant le temps, pourrait-on espérer la suivre? L'analyse par George Thomson du verbe hupokrinesthai, "jouer un rôle", pourrait nous aider à cerner le moment décisif où s'installe cette passivité. Comme Thomson le fait remarquer, hupokrinesthai signifie deux choses distinctes dans les poèmes homériques: "répondre" et "interpréter" (un présage ou un songe) ${ }^{14}$. Contrairement à d'autres savants, qui ont cherché à choisir entre ces deux significations pour expliquer l'origine de l'hupokritês, "acteur", Thomson se demande pourquoi elles ont été couvertes par un seul mot, comme dans un passage de l'Odyssée lorsque Peisistratos dit à Ménélas : "Explique [...] si c'est pour nous ou pour toi seul qu'un dieu fit voir ce présage !" Homère poursuit : "A ces mots, Ménélas [...] réfléchit afin de lui donner la réponse [hupokrinesthai] qui convenait." On aurait pu traduire également : "afin de lui donner l'interprétation qui convenait 15 ". La clé du problème, selon Thomson, est fournie par un passage du Timée, où il est dit "que les prophêtai sont bien des hupokritai de paroles et de signes énigmatiques, mais qu'ils ne sont nullement des mantai [des devins proférant leurs paroles en extase] ${ }^{16}$ ". Thomson conclut : hupokritês est à l'origine la désignation d'un personnage à qui l'on pose des questions concernant "des paroles et des signes énigmatiques" - son interprétation constituera sa réponse. Si ce personnage se trouve à la tête d'un chœur qui accomplit un rite dont la signification échappe à ceux qui y assistent, l'hupokritês peut "répondre" aux questions, en "interprétant" ce qui se passe, par exemple en disant : "Je suis Dionysos et celles-ci sont les filles d'Eleuthère que j'ai frappées de folie." Et plus tard, lorsqu'il commence à donner des "réponses-interprétations" sans qu'on les lui demande, il n'est du coup plus un hupokritês au sens ancien. Il est par là même devenu acteur. La séparation entre l'espace scénique (désormais autonome) et les spectateurs (désormais passifs) s'est installée. 
Or, c'est précisément le verbe hupokrinesthai, ou plutôt hupokrinomai, “je réponds", qui se lit dans l'inscription d'Andron fils d'Antiphanès, sur laquelle il convient maintenant de se pencher. Trouvée à Athènes, cette inscription métrique, en dialecte attique, appartenait à une statuette de bronze maintenant perdue, datant de la fin du VI ${ }^{\mathrm{e}}$ siècle avant J.-C. ${ }^{17}$ :

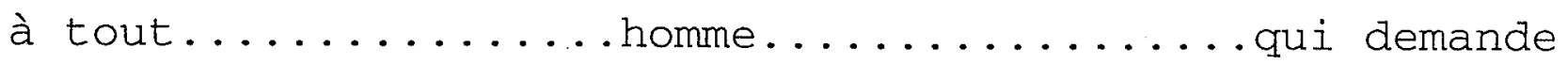

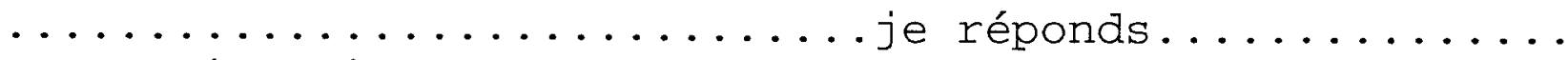
.......identiquement.

\section{hôs m' anethêk' Andrôn Antiphanous} dekatên

qu'...$\ldots \ldots \ldots$ Andron fils d'Antiphanès..........

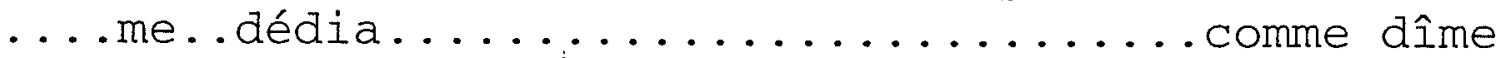

Sur l'inscription qui se transcrit et qui se traduit ainsi quelques remarques s'imposent. A la fin du $\mathrm{VI}^{\mathrm{e}}$ siècle, le théâtre existe déjà sous sa forme institutionnalisée : les concours commencent en 534 et les représentations tragiques - avant Eschyle avec un seul acteur et un chœur - remontent probablement à environ trente ans plus tôt ${ }^{18}$. Lorsque la statuette reçoit son inscription, le poète tragique Thespis (l'inventeur de l'acteur) est déjà en pleine activité. Le verbe hupokrinomai a donc inévitablement une signification plus riche que ne le laisse deviner ma traduction — "je réponds". En dialecte attique, "répondre" n'est pas en effet hupo-krinesthai comme en ionien. A Athènes, on emploie apo-krinesthai dans ce sens. Si l'auteur de l'inscription avait voulu écrire "je réponds", il aurait utilisé apokrinomai, qui est l'équivalent métrique de hupokrinomai. Il ne l'a pas fait. On est donc amené à croire que ce verbe a été choisi pour exprimer plus que la simple idée d'une réponse.

En employant hupokrinomai, la statuette inscrite élève sa "voix". Elle "parle". Et par la force des circonstances, son dire est donc un dire théâtral autant que vocal : par sa voix métaphorique, l'inscription répond à une question qui ne lui est pas posée, mais qu'elle anticipe, en toute autonomie. Tout comme l'hupokritês au théâtre, donnant sa réponse sans qu'on la lui demande.

Mais si hupokrinomai signifie en même temps qu'elle interprète ce qui est posé comme une énigme - à savoir : quel sens faut-il donner à la statuette inscrite ?-, elle s'interprète elle-même, elle se déchiffre devant les yeux du spectateur-lecteur, qui n'a pas à faire d'effort pour vocaliser l'écrit, puisque l'écrit se "vocalise" lui-même ici. Hypocrite lectrice, nous offrant la représentation de la voix ! En première, à vrai dire. Car avant l'invention de la lecture silencieuse, l'écriture visait la production d'une voix, non pas sa représentation. Jusqu'à sa mise en son, elle ne représentait pas plus que, pour nous, des lettres tapées par un singe dactylographe. 
S'adressant au spectateur-lecteur, qui ne doit pas faire entendre sa voix à lui, l'inscription peut désormais livrer son sens directement à l'œil : pourquoi lire à haute voix, si l'inscription sait "parler" en silence ? Le sens de l'objet arrive à l'œil du lecteur comme par une espèce d'irradiation ou d' "effluve". L'objet rayonne son sens sur le lecteur. Le sens de l'objet n'est plus laborieusement activé par la voix du lecteur. Son écriture est autonome, elle "parle". Telle est, me semble-t-il, la logique de cette inscription, témoignant de façon directe (et non pas, comme les passages de l'Hippolyte et des Cavaliers, de façon indirecte) d'une pratique de lecture silencieuse pour l'Athènes du VI' siècle finissant et, du même geste, de l'intériorisation de l'espace théâtral dans l'espace écrit. Désormais, l'espace scriptural est susceptible d'être une scène.

Si la statue dédiée par Andron est isolée en tant qu'objet "parlant", au sens strict de cette expression, le $V^{\mathrm{e}}$ siècle va rendre sa métaphore de plus en plus courante. Non pas tant dans le domaine des inscriptions, mais chez les auteurs qui pratiquent une écriture moins laconique et qui, pour cette raison, sont plus susceptibles de changer leurs habitudes de lecture.

Mon premier exemple est emprunté à Eschyle, dont la précédence dans ce domaine est très significative (on comprendra bientôt pourquoi). Chez lui, l'emploi de la métaphore est suggéré par trois boucliers de héros, à savoir ceux de Capanée, d'Etéocle et de Polynice dans les Sept contre Thèbes. "Pour blason, dit le Messager à Etéocle, Capanée a un homme nu, portant le feu; une torche flambante arme ses mains, et il proclame [phônei] en lettres d'or : 'J'incendierai la ville ${ }^{19}$." Dans une pièce de théâtre où l'on rencontre l'expression synesthétique remarquable "je vois le fracas ${ }^{20}$, , il semble logique que les objets prennent la parole et que le personnage dessiné sur le bouclier "vocifère", comme sur le bouclier cité, ou bien "crie", boai, comme sur le bouclier d'Etéocle, à travers des lettres alphabétiques dessinées à côté de lui ${ }^{21}$. Sur le bouclier de Polynice, finalement, on voit la Justice personnifiée, identifiée non pas grâce à ses attributs traditionnels mais grâce à une légende : "Et celle-ci se prétend la Justice, comme le dit [legei] l'inscription placée près d'elle ${ }^{22}$."

Mon deuxième exemple est tiré d'Hérodote. Chez lui, les lettres de l'alphabet commencent également à parler, legein, et cela massivement ${ }^{23}$; et les oracles écrits, les stèles et les tripodes élèvent eux aussi la "voix ${ }^{24 " ~ a i n s i ~ q u e ~ l a ~ s t a t u e ~ d e ~ p i e r r e ~ d u ~}$ roi égyptien Séthos, qui "prononce" sa propre inscription ${ }^{25}$. Pour l'historien qui écrit de façon extensive et qui lit encore plus, la lecture silencieuse, rendue mentalement possible par l'expérience du théâtre (on peut rappeler ici qu'Hérodote est l'ami de Sophocle), s'impose naturellement. Il a besoin de lire vite, ne serait-ce que pour mieux élaborer sa propre œuvre écrite. Et accélérer la vitesse de la lecture signifie à un certain point nécessairement intérioriser la voix lectrice. Faire abstraction de la voix et lire de tête.

L'inscription d'Andron fils d'Antiphanès marque un moment décisif dans les rapports des Grecs avec l'espace écrit : ce n'est pas un hasard si le Phèdre de Platon lui fait écho, à plus d'un siècle de distance, dans un passage concernant le propre de l'écriture ${ }^{26}$. Comparant l'écriture à la peinture, Socrate reproche à l'écrit de "signifier toujours la même chose", c'est-à-dire cela même dont l'inscription d'Andron se vante. Naturellement, le philosophe aurait pu adresser le même repro- 
che à un acteur, dont la voix n'est que l'instrument d'un texte immuable et non pas celle de quelqu'un en possession du savoir, epistêmê. En effet, il le fait, ailleurs ${ }^{27}$. Les deux choses reviennent au même car, comme on l'a vu, l'écrit et l'acteur sont analogues, interchangeables. L'acteur se substitue à l'écrit sur la scène, l'écrit se substitue à l'acteur dans l'inscription d'Andron. Produisant ce que j'appelle une "écriture vocale", l'acteur ouvre la possibilité d'une nouvelle attitude vis-à-vis de l'écrit, la possibilité d'une lecture silencieuse. En fait, la statuette inscrite dédiée par Andron se définit comme "acteur", hupokritês, ce qui présuppose cette nouvelle attitude. L'espace écrit est une "scène" qui emprunte sa logique au spectacle théâtral, en attribuant le rôle du spectateur au lecteur. Autrement dit, l'espace écrit intériorise le théâtre.

Cette conclusion est justifiée à la fois par l'inscription d'Andron et par un passage comme celui de l'Hippolyte d'Euripide, déjà cité, où la "tablette d'écriture" de Phèdre morte "crie, crie des horreurs [boai boai deltos alasta] ${ }^{28 "}$ ". Tel qu'il est mis en scène par Euripide, l'écrit est censé non seulement "parler" ${ }^{29 "}$ " pendant l'acte de la lecture silencieuse mais encore "crier". Il est même capable de de chanter : "Tel est, tel est le chant sonore - continue Thésée quelques vers plus loin - que j'ai vu s'élever de ces lignes écrites [hoion hoion eidon en graphais melos phthengomenon] ${ }^{30}$." L'acteur qui chante le rôle de Thésée (nous sommes dans un passage lyrique) chante donc d'un melos sonore qui surgit de l'écrit, c'est-à-dire : d'un chant pour l'œil.

Sur la scène, un acteur qui chante; sur la tablette d'écriture - lue en silence et, par là même, intériorisant l'espace théâtral — des lettres qui "chantent". Il est difficile d'imaginer une mise en scène plus instructive de la lecture silencieuse que celle-ci. Et cela pour deux raisons. D'abord, elle fait intervenir, dans un chant au premier degré livré depuis la scène, le chant figuré de l'écrit, soulignant, par cette inclusion, l'analogie entre l'espace théâtral et l'écrit lu en silence. Ensuite, elle établit clairement la corrélation entre l'objet "parlant" et la lecture silencieuse : à la "voix" entendue dans la tête au cours de la lecture silencieuse correspond précisément l'objet "parlant".

Le témoignage de l' Hippolyte ne se réduit donc pas aux faits externes - qui ne permettent pas de distinguer de façon indiscutable entre lecture silencieuse et lecture simplement inaudible pour autrui -, mais il comporte un aspect interne, qui corrobore l'interprétation de Bernard Knox, en y ajoutant des faits se rapportant à l'architecture mentale d'une lecture vraiment silencieuse.

$\mathrm{Si}$, de cette manière, le théâtre s'intériorise dans le livre, le livre s'intériorise à son tour dans l'espace mental, tantôt désigné comme phrên, tantôt comme psukhê. Et cela bien avant Platon, qui dans un passage du Phèdre oppose l'écriture ordinaire à la fameuse "écriture dans l'âme ${ }^{31}$." Notre premier témoin de la métaphore "le livre de l'âme" est en effet Pindare, qui dans une ode datant de 474 av. J.-C. s'exclame : "Lisez-moi le nom du vainqueur olympique, là où il est écrit dans mon esprit [phrên] ! ${ }^{32 ", ~}$

Mais c'est chez les tragiques que cette métaphore connaîtra sa plus grande fortune avant d'être reprise par Platon. Et pour cause : les poètes dramatiques, qui produisent des textes destinés à être appris par cœur par leurs acteurs, vivent très 
concrètement l'inscription du texte dans l'esprit de l'acteur. Pour le poète dramatique, l'acteur reçoit une inscription, autant que telle pierre ou feuille d'écriture. L'intérieur de l'acteur est un espace scriptural. Ce qui veut dire que le texte dramatique est "inscrit" dans l'esprit de celui qui le dit sur scène.

Ainsi se justifie l'expression "écriture vocale" utilisée ici à plusieurs reprises, et l'on comprend pourquoi Eschyle - qui introduit le deuxième acteur ${ }^{33}$ - écrit dans la mémoire de ses acteurs, tandis qu'un Homère (fût-il scripteur) ne peut pas être considéré comme quelqu'un qui écrit dans la mémoire de ses récitants, trop séparés de lui - dans le temps et dans l'espace - pour qu'une telle métaphore puisse être pertinente.

Citons les exemples tirés de l'œuvre d'Eschyle, bien que la même métaphore revienne chez les deux autres grands tragiques. Dans Prométhée enchaîné, le protagoniste déclare : "A toi d'abord, Io, je dirai les erreurs de ta course tourbillonnante : inscris-les sur les tablettes fidèles de ta mémoire ! ${ }^{34 "}$ Prométhée est lié à l'origine de l'écriture; selon une tradition, Danaos l'est aussi. Voici comment il s'adresse à ses filles dans les Suppliantes: "Et maintenant, à terre, ma prévoyance encore vous engage à garder mes avis bien gravés en vous" 35 . Et la métaphore revient plus loin dans la même pièce lorsqu'il dit : "Maintenant, aux nombreuses leçons de modestie inscrites en vous par votre père, vous ajouterez l'inscription suivante : '[... $]^{36 " \text { ". }}$ Dans les Euménides, le chœur compare la mémoire de Hadès à une tablette d'écriture: "Hadès, sous terre, exige des humains de terribles comptes, et son âme [phrên] qui voit tout, de tout garde fidèle transcription ${ }^{37}$." Et dans mon dernier exemple, Electre dit à Oreste : "Entends et inscris dans ton cœur [phrenes] ${ }^{38}$." C'est une formule que le poète tragique aurait pu utiliser lui-même en s'adressant à l'un de ses acteurs.

Jesper SVENBRO

C.N.R.S

1. Ce texte reprend, en le résumant, un chapitre de mon livre Phrasikleia. Anthropologie de la lecture en Grèce ancienne, Paris, La Découverte, 1988, pp. 178-206 ("La voix intérieure. Sur l'invention de la lecture silencieuse"); voir aussi "La Grecia arcaica e classica: l'invenzione della lettura silenziosa", dans R. Chartier et G. Cavallo (ed.), Storia della lettura nel mondo occidentale, Rome-Bari, Laterza, 1995, pp. 3-36.

2. B.M.W. Knox, "Silent Reading in Antiquity", Greek, Roman and Byzantine Studies, 9, 1968, pp. 421-35.

3. Euripide, Hippolyte, 856-86.

4. Aristophane, Cavaliers, 115 sq. 
5. Voir Knox, art. cité, pp. 421 et 435.

6. Cf. Plutarque, Vie de Lycurge, 16,10.

7. Voir Phrasikleia, op. cit., p. 72-73, etc.

8. P. Saenger, "Silent Reading: Its Impact on Late Medieval Script and Society", Viator, 13, 1982, p. 378.

9. Knox, art. cité, pp. 421-422.

10. Saenger, art. cité, pp. 378-80; 383-84; 405.

11. Si les poètes comiques semblent parfois ignorer l'existence de cette séparation, c'est que sa transgression produit un effet comique : la séparation est donc bel et bien là. Pour la façon rigoureuse dont les tragiques ont respecté la démarcation entre l'espace fictif et le public, voir D. Bain, "Audience Address in Greek Tragedy", Classical Quarterly, 25, 1975, pp. 13-25.

12. Plutarque, Vie de Solon, 29.

13. Cf. Ch. Segal, La Musique du Sphinx. Poésie et structure dans la tragédie grecque, Paris, La Découverte, 1987, pp. 263-98.

14. G. Thomson, Aeschylus and Athens, $2^{\mathrm{e}}$ éd., Londres, Lawrence \& Wishart, 1950, pp. 181-82.

15. Odyssée, 15,167-70.

16. Platon, Timée, 72a-b.

17. Inscriptiones Graecae, $\mathrm{I}^{2}, 410\left(=\mathrm{n}^{\circ} 658\right.$ Lazzarini).

18. A. Pickard-Cambridge, Dithyramb, Tragedy and Comedy, $2^{\mathrm{e}}$ éd., Oxford U. P., 1962, p. 88.

19. Eschyle, Les Sept, 432-34.

20. Ibid., 103.

21. Ibid., 465-69. 


\section{LA LECTURE SILENCIEUSE}

22. Ibid., 646-48; cf. 660 .

23. Hérodote, I, 124, 187; II,106, 136; III,88; IV,91; VII,228; VIII,22.

24. Idem, II,133; V,90, 92; VI,77; VIII,136; II,102; V,60, 61.

25. Idem, II, 141 .

26. Platon, Phèdre, 275d.

27. Platon, Ion, 532d; etc.

28. Euripide, Hippolyte, 877.

29. Euripide, Iphigénie en Tauride, 641-42; 584-85.

30. Euripide, Hippolyte, 879-80.

31. Platon, Phèdre, 275d-276a; cf. Philèbe, 38e-39a.

32. Pindare, Olympiques, 10,1-3.

33. Aristote, Poétique, 4,1449a16.

34. Eschyle, Prométhée enchaîné, 788-89.

35. Eschyle, Suppliantes, 178-89.

36. Ibid., 991-92.

37. Eschyle, Euménides, 273-75.

38. Eschyle, Choéphores, 450. 